\title{
Developing a Compact Particle Collector by Integrating a Wet Electrostatic Precipitator and an Inertia Mist Eliminator
}

\author{
Zhengda Yang ${ }^{1,2}$, Hongbo Qiu ${ }^{3}$, Can Zhou ${ }^{1}$, Ye Jiang ${ }^{2}$, Riyi Lin ${ }^{2}$, Yang Yang ${ }^{1 *}$, \\ Chenghang Zheng ${ }^{1}$, Xiang Gao $^{1}$
}

${ }^{1}$ State Key Lab of Clean Energy Utilization, Zhejiang University, Hangzhou 310027, China

${ }^{2}$ College of New Energy, Qingdao Engineering Research Center of Efficient and Clean Utilization of Fossil Energy, China University of Petroleum (East China), Qingdao 266580, China

${ }^{3}$ Shandong Guoshun Construction Group Co., Ltd., Jinan 250000, China

\begin{abstract}
Wet electrostatic precipitator (WESP) is a reliable alternative to realize the ultra-low emission of particulate matter from coal-fired power plants. This work proposes to develop a compact particle collector by integrating a WESP and an inertia mist eliminator (IME). The particle collection efficiency and charging characteristics of the WESP were analyzed to determine the potential for compact design. Results show that the WESP was easy to achieve a collection efficiency of $95 \%$ for both pin and wire electrodes. Particle charging can still be guaranteed with short WESP length and high voltage, indicating the particle charging was sensitive to electrical parameters. Charges carried by the escaping particles acted as a bridge between the WESP and the IME. The collision enhanced by particle charging can lead to an improvement in overall efficiency and charge loss. The particle charge loss of the particle sized $7.29 \mu \mathrm{m}$ was $22.6 \%$ and $29.4 \%$ for the one and two stage IME, which coincided with the efficiency enhancement of WESP. The collection efficiency significantly decreased as the WESP size decreased, but the collection efficiency can be enhanced by the combination with IME. The 6-wire WESP enhanced by one-stage IME can be considered the optimal combination because the WESP size can be shorten by $40 \%$ while maintaining a similar efficiency. The research findings are beneficial for the retrofit of air pollution control devices in limited space.
\end{abstract}

Keywords: Particulate matter, Ultra-low emission, Wet electrostatic precipitator, Mist eliminator, Device retrofit

Revised: September 30, 2021

Accepted: October 6, 2021

${ }^{*}$ Corresponding Author:

11527077@zju.edu.cn

Publisher:

Taiwan Association for Aerosol Research

ISSN: $1680-8584$ print

ISSN: 2071-1409 online

Copyright: The Author(s). This is an open access article distributed under the terms of the Creative Commons Attribution License (CC BY 4.0), which permits unrestricted use, distribution, and reproduction in any medium, provided the original author and source are cited.

\section{INTRODUCTION}

The pollutants of sulfur dioxide, nitrogen oxides, particulate matter (PM) discharged from energy utilization and conversion processes are crucial for the severe air pollution in recent years (Gao et al., 2018; Jiang et al., 2021; Ye et al., 2020). Recently, much attention is paid to particulate pollution because of the popularity of the $\mathrm{PM}_{2.5}$ concept (particle with diameter less than 2.5 micrometers) (Chen et al., 2019). Therefore, the PM emission standards become strict around the world. In China, the PM in flue gases from coal-fired power plants and steel sintering is required lower than $5 \mathrm{mg} \mathrm{m}^{-3}$ according to the latest ultralow emission standard (Cui et al., 2018; Yang et al., 2021).

Various dust removal technologies based on inertia, filterable, and electrostatic mechanisms have been developed to eliminate the PM emission. Low-low temperature electrostatic precipitator (LLTESP), electrostatic fabric filter (EFF), and wet electrostatic precipitator (WESP) are applied to realize the PM ultra-low emission (Li et al., 2019; Yang et al., 2019). The PM emission concentration can be reduced below $10 \mathrm{mg} \mathrm{m}^{-3}$ with LLTESPs and EFFs and even below $5 \mathrm{mg} \mathrm{m}^{-3}$ with the further 
assistance of highly efficient demisters equipped at the wet flue gas desulfurization tower (WFGD) (Huang et al., 2020). However, the stability of ultralow emission under such conditions strongly depends on the demister performance (Liang et al., 2020). WESP is typically arranged at downstream of the WFGD to remove the slurry droplets, fly ashes, and acid mists with an overall efficiency $>90 \%$ (Zheng et al., 2019). However, additional installation space is needed to install WESP and thus WESPs are suitable for new units or existing units but with adequate retrofit space (Xu et al., 2016). Notably, the application scope of WESP can be broadened if the WESP size can be reduced.

Generally, the WESP size can be reduced if the collection efficiency can be guaranteed with a small size. Agglomeration methods, including electrical agglomeration (Chang et al., 2017), acoustic agglomeration (Zhou et al., 2016), turbulence agglomeration (Shuangchen et al., 2016), and chemical agglomeration (Liu et al., 2016), have been developed to improve particle collection efficiency by enlarging particle size. Although these technologies can enhance the particle collection without additional space, the whole control system can be very complicated. Moreover, they are mainly proven promising in laboratory research, and few are put into use in practical applications. Previously, our research group developed a combined WESP by integrating a pre-charger with a conventional WESP (Yang et al., 2018a). The specific collection area of the combined WESP could be reduced by $22.7 \%$ with a similar collection efficiency. However, this improvement still cannot fulfill the compact requirement for WESP installation in limited space. Fortunately, the WESP itself, according to the previous results, can be treated as a charger as well because the escaping particle carried a high number of charges (Yang et al., 2018b).

The interaction characteristics between particle and wall surface were different for charged and neutral particles ( $\mathrm{Li}$ and Marshall, 2007). The particle precipitation condition on the wall surface may be changed after partricle charging (Krupa et al., 2019). Some researchers attempted to take advantage of this property to improve the inertial separation efficiency, thereby developing the electrostatic cyclone (Wang et al., 2020). The cyclone dust collector is not suitable for combination with WESP. Nevertheless, the plate-type mist eliminator also uses inertia to achieve the collision and capture of particles on plates in applications (Song and $\mathrm{Hu}, 2017$ ). It benefits from the flat plate structure with a small overall size (thickness), which can satisfy the compact requirement. However, the feasibility of the combination of the two and the optimal combination is still not understood.

Aiming at the compact design of WESP, we propose to integrate the WESP with an inertia mist eliminator (IME) to make full use of the charging property of escaping particles. The performance of the WESP was analyzed to determine the potential for compact design. The charge amount carried by escaping particles was investigated under various operating voltage and charging time. Then, the particle collection efficiency and charge loss were analyzed under different combinations of WESP and IME. Finally, the collection performance under different WESP sizes and IME combinations was evaluated, and the optimal combination scheme was determined as a consequence.

\section{EXPERIMENTAL METHODS}

As exhibited in Fig. 1, a schematic of the gas purification system was established for evaluating particle collection performance of the WESP, IME, and their combinations. The WESP and IME were arranged after a WFGD tower. The sub-sections (including a air fan, a particle feeder, and WFGD) ahead of the WESP simulate the wet flue gas contaminated with fine particles. A vibratory particle feeder was used to disperse particles with a stable feeding rate, and the test particle was collected from the ash hopper in a coal-fired power station. Detailed information has been introduced in previous work (Yang et al., 2017). The overall gas flow rate was controlled within 96-132 $\mathrm{m}^{-3} \mathrm{~h}$, corresponding to a residence time of 1.18-1.62 s.

The WESP was horizontally arranged with a $120 \mathrm{~mm} \times 300 \mathrm{~mm}$ cross-section and a $1200 \mathrm{~mm}$ length. Discharge electrodes were placed in the center of the WESP channel, and thus the electrode-plate space was $60 \mathrm{~mm}$. Initially, the WESP was configured with 10 electrodes with an interval of $100 \mathrm{~mm}$. Two types of electrodes, including wire and pin electrodes, were investigated. The latter was further classified into vertical and parallel configurations, labeled as pin-v and pin$p$, according to the direction between the pin and gas flow. The geometries schematic of the pin electrode have been introduced in the previous work (Yang et al., 2017). When the electrode 


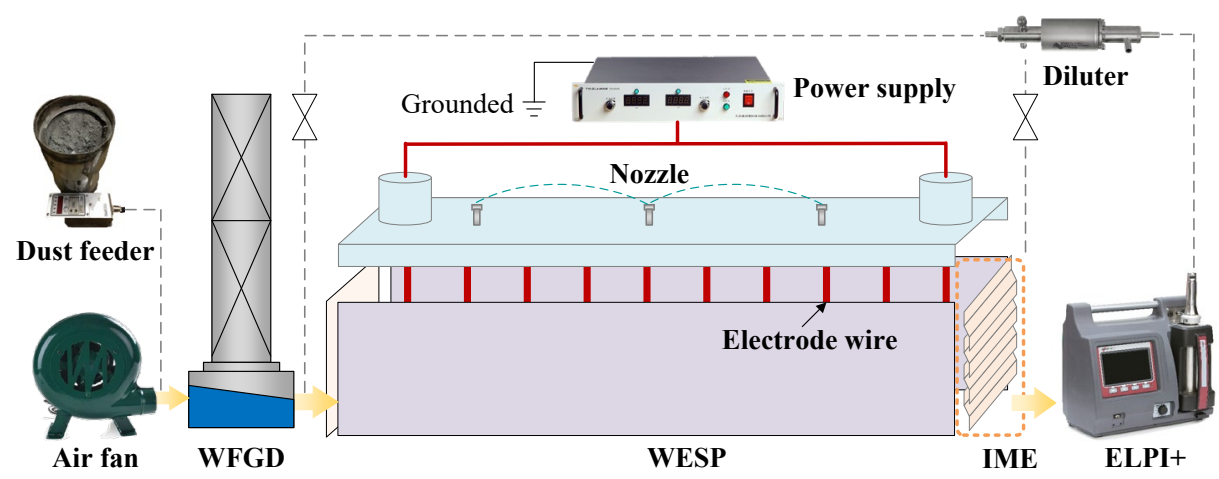

Fig. 1. Schematic of the gas purification system.

number was reduced, the effective length of WESP was shortened as well. The discharge electrode was connected with a high-frequency power source with a frequency of $20 \mathrm{kHz}$. In this study, the power source supplied a operation voltage in the range of 10-30 kV. As shown in the dotted section in Fig. 1, an inertia mist eliminator was installed at the outlet of the WESP to inhibit the escape of mist droplets. The mist were originated from the droplet entrainment of spraying in WFGD and WESP, respectively. The droplet entrainment of the former cannot be avoided since WFGD operated continuously. By contrast, the droplet entrainment of WESP can be minimized because the spraying in WESP worked intermittently. In this study, the inertia mist eliminator was designed with a deflection plate type. The curvature of the bend in the streamline is of vital importance to the mist impaction probability on the plate surface. Therefore, the geometry of the mist eliminator was modified through flow field simulation, and the geometry details are shown in Fig. 2(a). Moreover, the inertia mist eliminator can be doubled into two-stage structure to further improve its performance. The geometry details and photos of the two-stage IME are shown in Figs. 2(b) and 2(c), respectively.

The particle concentration was continuously monitored with an electrostatic low-pressure impactor (ELPI ${ }^{+}$, Dekati Ltd., Finland). The ELPI ${ }^{+}$works on the principle of aerodynamics impact and induced current to classify particle size and number concentration. Its work process has been introduced more than once in previous work, so more detailed information can be found in those papers (Yang et al., 2020a). The flue gas can be sampled both from the outlet and inlet of the WESP by switching the sampling pipe. Since the WESP operated under wet flue gas conditions, the humid sampling gas may lead to the charger's unfavorable insulation deterioration in the $\mathrm{ELPI}^{+}$. The sampled flue gas was diluted by an ejection diluter (Diluter DI-1000, Dekati Ltd., Finland) with a ratio of 8 to avoid the insulation deterioration. The sampled flow rate after dilution was $10 \mathrm{~L} \mathrm{~min}^{-1}$ in total. Particles were charged to known charge amounts in the charger of the ELPI ${ }^{+}$. The charged particle can subsequently induce a current in the impactors of the ELPI ${ }^{+}$. The current signals were detected to calculate particle concentration. Fig. 3 shows the size distribution of the tested particle obtained by the ELPI ${ }^{+}$.

All tests followed a similar procedure to evaluate the particle collection performance: (1) Operating and stabilizing the gas purification system; (2) Measuring the particle concentration at the inlet; (3) Measuring the particle concentration or charge amount at the outlet; (4) Switching test conditions and repeating (1)-(3). All tests were carried out when the particle concentration fluctuated within $\pm 5 \%$. The particle collection efficiency was calculated with concentration data averaged over one minute, as shown in Eq. (1).

$\eta=\left(1-\overline{c_{o}} / \overline{c_{i}}\right) \times 100 \%$

where $\eta$ is the collection efficiency (\%), $\overline{c_{o}}$ and $\overline{c_{i}}$ represent the averaged particle concentration $\left(\mathrm{mg} \mathrm{m}^{-3}\right)$ at the outlet and inlet of the WESP, respectively.

The particle charge amount can be converted from the signal of the induced current of ELPI with Eq. (2). Note that the pre-charger of $\mathrm{ELPI}^{+}$should be turned off to ensure all particle charges were obtained from the WESP. 


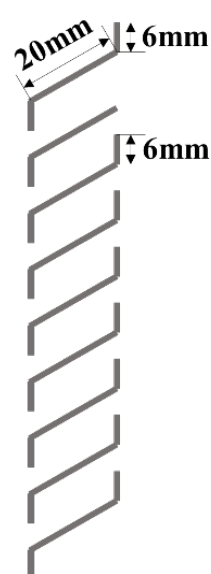

(a)

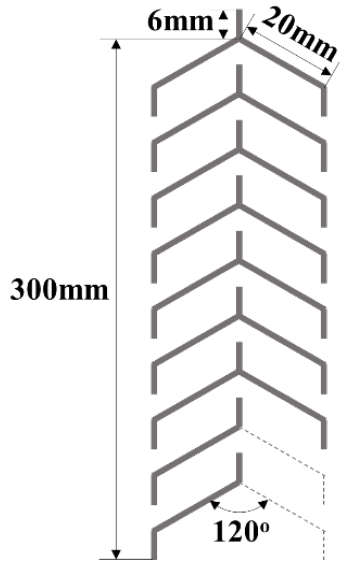

(b)

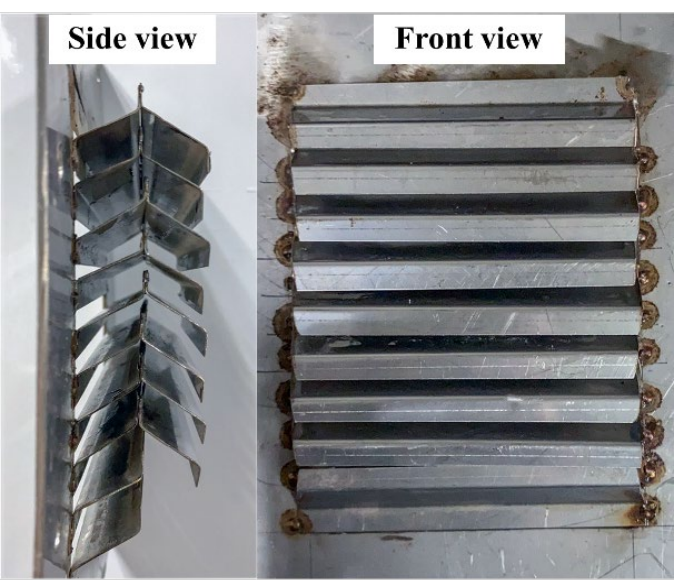

(c)

Fig. 2. Geometries schematic of (a) one-stage IME, (b) two-stage IME, and (c) photos of two-stage IME.

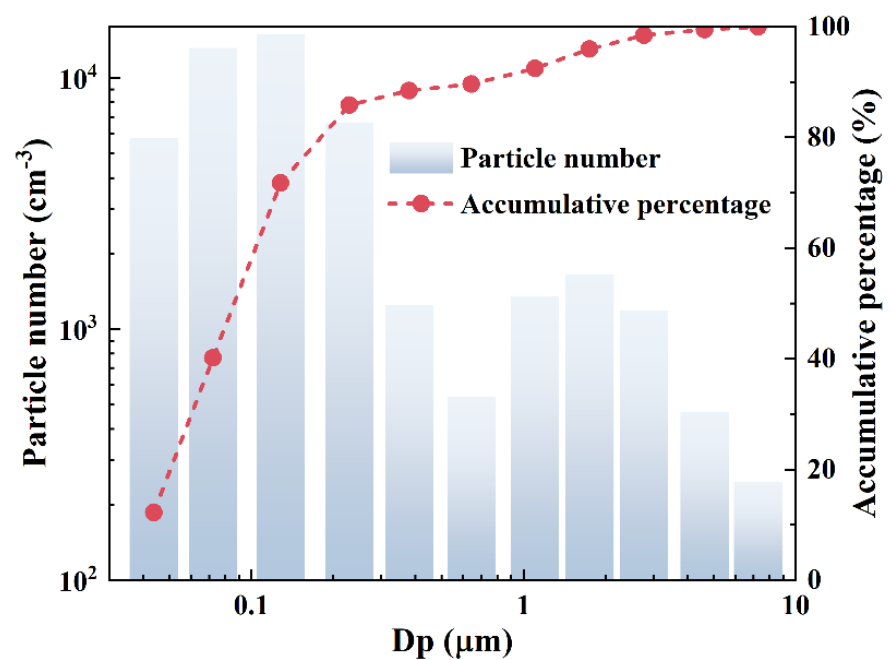

Fig. 3. Size distribution of the tested particle.

$n=\frac{I_{i}}{Q \times N \times e}$

where $n$ is the particle charge amount, $l_{i}$ is the grade induced current of ELPI ${ }^{+}$with charger off $(\mathrm{A})$, $N$ is the grade particle number concentration $\left(\mathrm{cm}^{-3}\right), Q$ is the sampling flow rate $\left(\mathrm{cm}^{3} \mathrm{~s}^{-1}\right)$, and e is the elementary charge $\left(1.602 \times 10^{-19} \mathrm{C}\right)$. Here, the unit " $\mathrm{C}$ " is short for coulombs.

\section{RESULTS AND DISCUSSION}

\subsection{Performance of the WESP}

Prior to developing a novel particle collector, the performance of the WESP was evaluated in terms of discharge characteristics and collection efficiency. The I-V curves of different discharge electrodes are shown in Fig. 4(a). The I-V curves of all electrodes showed similar nonlinear tendencies. Compared with the wire electrode, the pin electrode of both vertical and parallel configurations presented higher coron intensity under the same operating voltage. Moreover, the corona current of the vertical configuration was higher than that of the parallel configuration under the same operating voltage. The pin electrode has lots of sharp surfaces, which are beneficial 

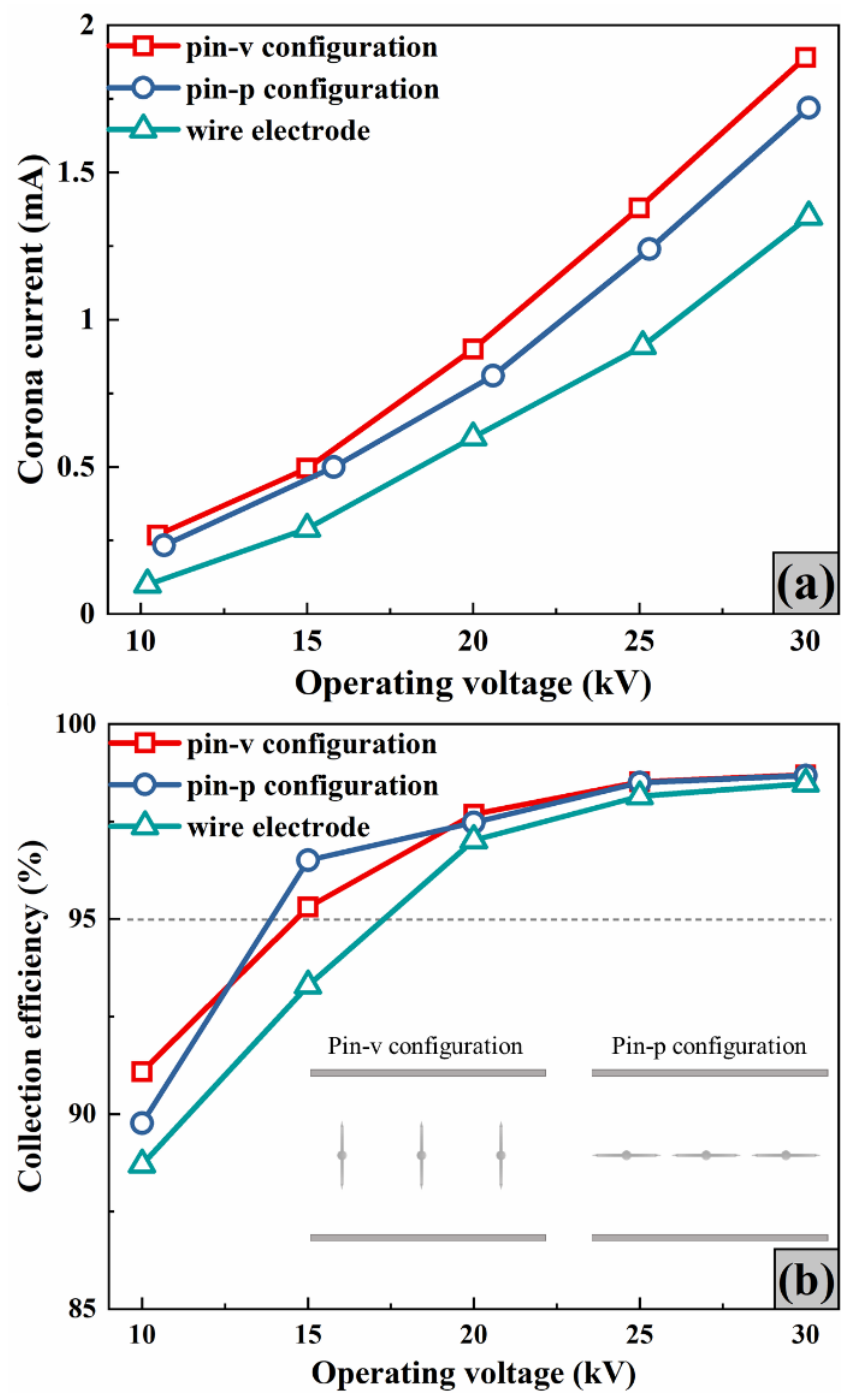

Fig. 4. Overall performance of (a) I-V characteristics and (b) collection efficiencies of various electrodes.

for corona discharge to occur at low voltages, according to Peek's formula (Yang et al., 2018c). The distance between the electrode edge and plate surface was reduced when the electrode configuration changed from pin-p to pin-v. The electric field strength increased as the distance decreased at a given operating voltage. Different discharge characteristics can lead to the difference in collection efficiency, and the collection efficiencies of different electrodes are shown in Fig. 4(b). As expected, the collection efficiency of the pin electrode was higher than the wire electrode, especially in the low voltage ranges. The phenomenon can be attributed to that the ion density generated by corona discharge was low, which was not sufficient for particle charging (Yang et al., 2018c). When the operating voltage increased above $24 \mathrm{kV}$, the efficiency difference of the wire and pin electrode became small. However, the efficiency difference of pin electrode with different configurations was not as evident as the current difference. The pin- $v$ configuration provided higher corona currents and stronger electric fields than the pin-p configuration, while each pin only covered one side of the discharge channel. By contrast, the pin of the pin-p configuration exerted the impacts on both sides. Namely, although the discharge density was a little lower, the pin-p configuration could cover more space than the pin-v one.

Although the overall collection efficiency was high, there were still some particles escaping from the WESP. The size distributions of escaping particles under various operating voltages are illustrated in Fig. 5(a). The tested particles showed a bimodal size distribution, with two peaks around $0.13 \mu \mathrm{m}$ and $1.75 \mu \mathrm{m}$. The number concentration decreased dramatically but kept a similar size distribution with the increasing voltage. Given the inlet as a baseline, the size distribution can 

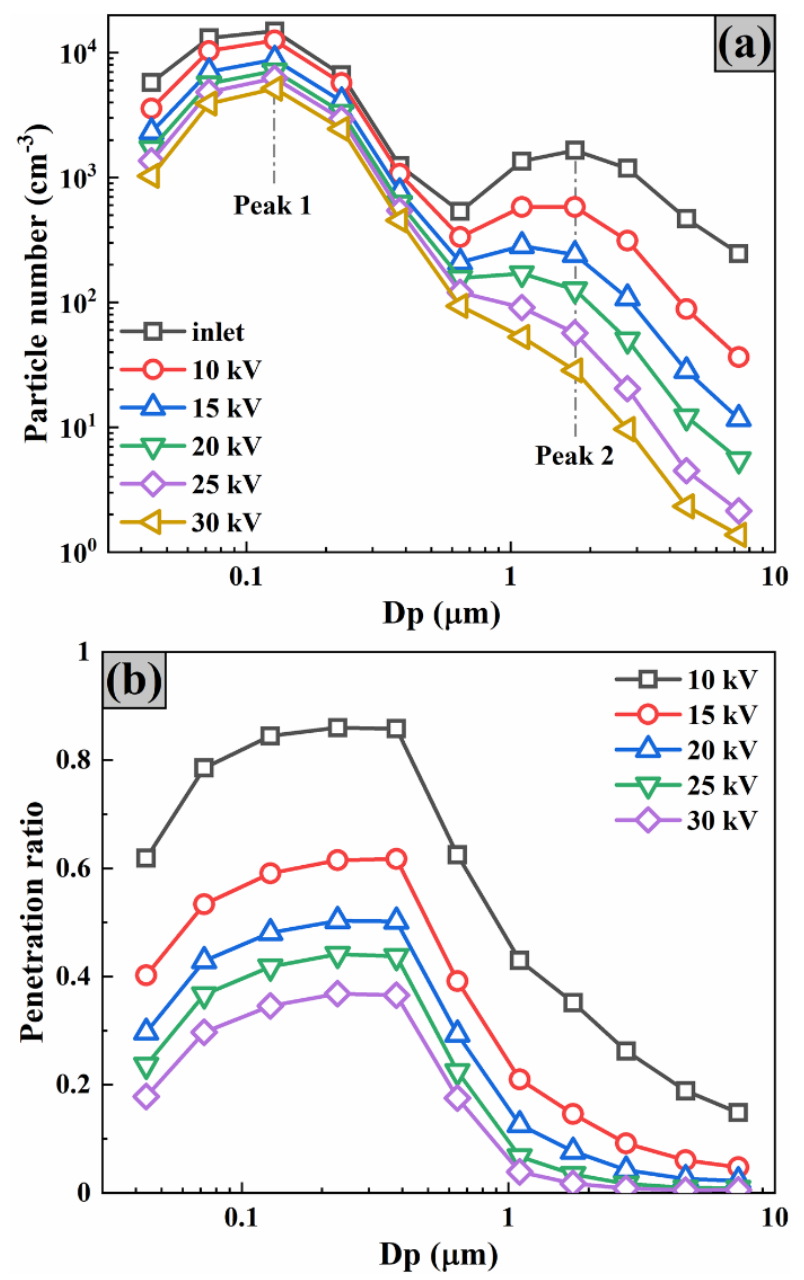

Fig. 5. (a) The size distribution, (b) penetration ratio of escaping particles under various operating voltage.

be converted into the penetration ratio, as shown in Fig. 5(b). The penetration ratio decreased with increasing voltage, whereas it reached a maximum around $0.38 \mu \mathrm{m}$ under all voltages. This can be ascribed to the minimal particle electrical mobility in this size range (Mizuno, 2000). However, particles around this range account for little proportion in the overall mass concentration. Even though the maximum penetration ratio was 0.37 at the voltage of $30 \mathrm{kV}$, the mass-based collection efficiency of all electrodes was above $95 \%$. Therefore, the WESP is easy to achieve very low emissions. This satisfactory performance provides the potential for developing a compact particle collector.

\subsection{Charges Carried by the Escaping Particles}

Insides a WESP, particles were electrically charged after passing through the ionic field and electric field. Most of the charged particles were separated from the flue gas, but some particles may still escape from the WESP, as discussed above. The escaping particles carried some charges (Huang and Chen, 2002). The charge amount of the escaping particle can be even higher than that of the collected ones because the former resided a long time and were close to the discharge electrode (Yang et al., 2020b). The charging characteristics of the escaping particles are fundamental for the coupling of WESP between other devices. The charge amounts carried by the escaping particles were investigated under various operating voltage and charging time.

\subsubsection{Effect of operating voltage}

The charge distribution of the escaping particle under different operating voltages is shown in 

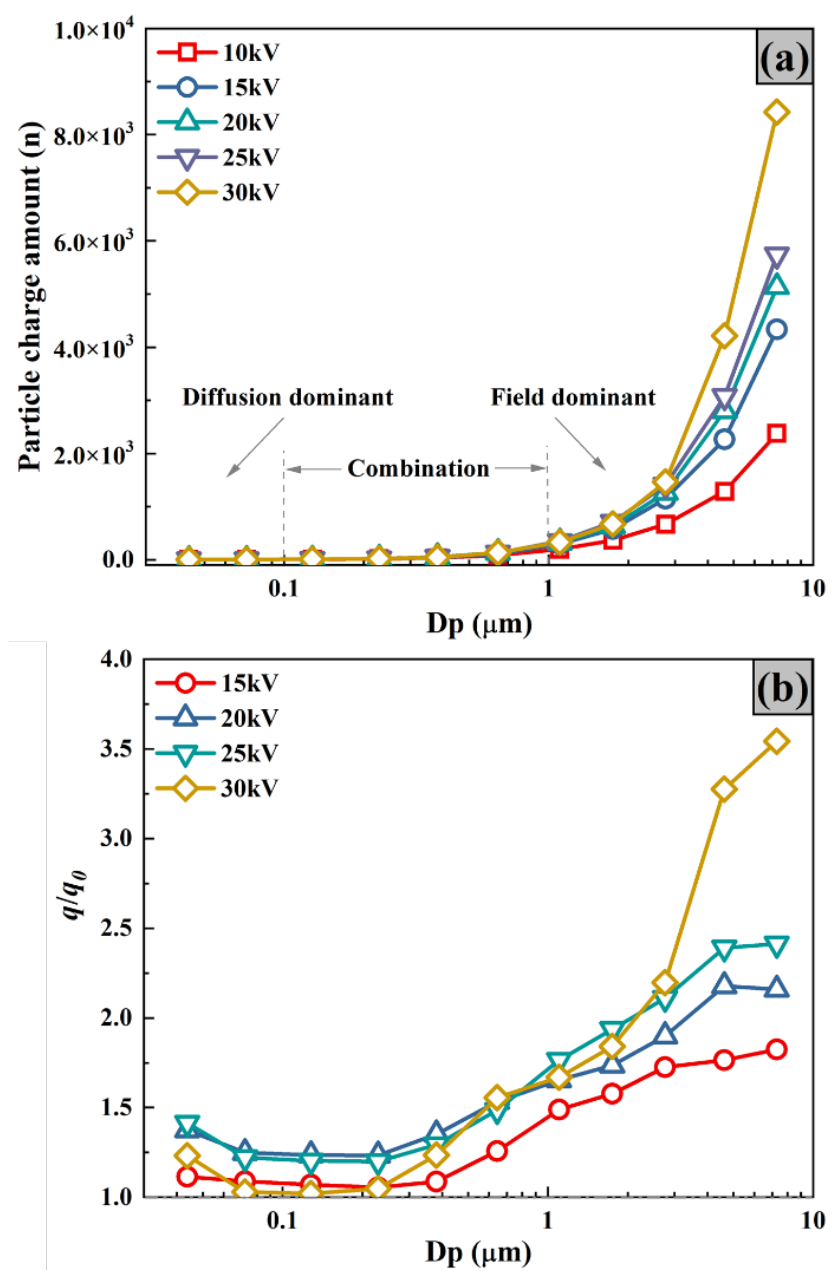

Fig. 6. Charge amount carried by grade particles under various operating voltages, (a) absolute charge amount, (b) relative charge amount.

Fig. 6(a) as a function of particle size. As discussed above, the pin-v electrode configuration was more beneficial for particle charging. Thus the charge amounts obtained in the subsequent investigations were all in this configuration. The results indicated that the charge amount increased by several orders with particle size, especially when the particle diameter exceeded $1.0 \mu \mathrm{m}$. Theoretically, the particle charging process is dominated by diffusion and field charging (Mizuno, 2000). Fig. 6(a) illustrated that the diffusion charging was the dominant factor of particle charging for particles $<0.1 \mu \mathrm{m}$, while the field charging dominated particle charging for particles $>1.0 \mu \mathrm{m}$. The charge amount was positively affected by the operating voltage. The maximum number of elementary charges for particle-sized $7.29 \mu \mathrm{m}$ was as high as 8428 when the operating voltage increased to $32 \mathrm{kV}$. The data obtained under a voltage of $10 \mathrm{kV}$ was set as a baseline $\left(q_{0}\right)$ to understand the enhancement by increasing voltage. Fig. $6(\mathrm{~b})$ indicated that the enhancement amplitude $\left(q / q_{0}\right)$ increased with the increasing voltage. Moreover, coarse particles were more sensitive to the operating voltage than fine particles. As the operating voltage increased, the ion density and electric field strength increased simultaneously, which mainly contributed to the diffusion and field charging, respectively (Lu et al., 2016; Mizuno, 2000). Therefore, the increase in particle charge was mainly attributed to the enhancement of field charging with the increasing voltage. Additionally, it can also be concluded that high operating voltage should be guaranteed when developing a miniaturized WESP.

\subsubsection{Effect of charging time}

Theoretically, the charging of submicron particles is more affected by diffusion charging than micron particles (Mizuno, 2000). The charge distribution of the escaping particle under various 

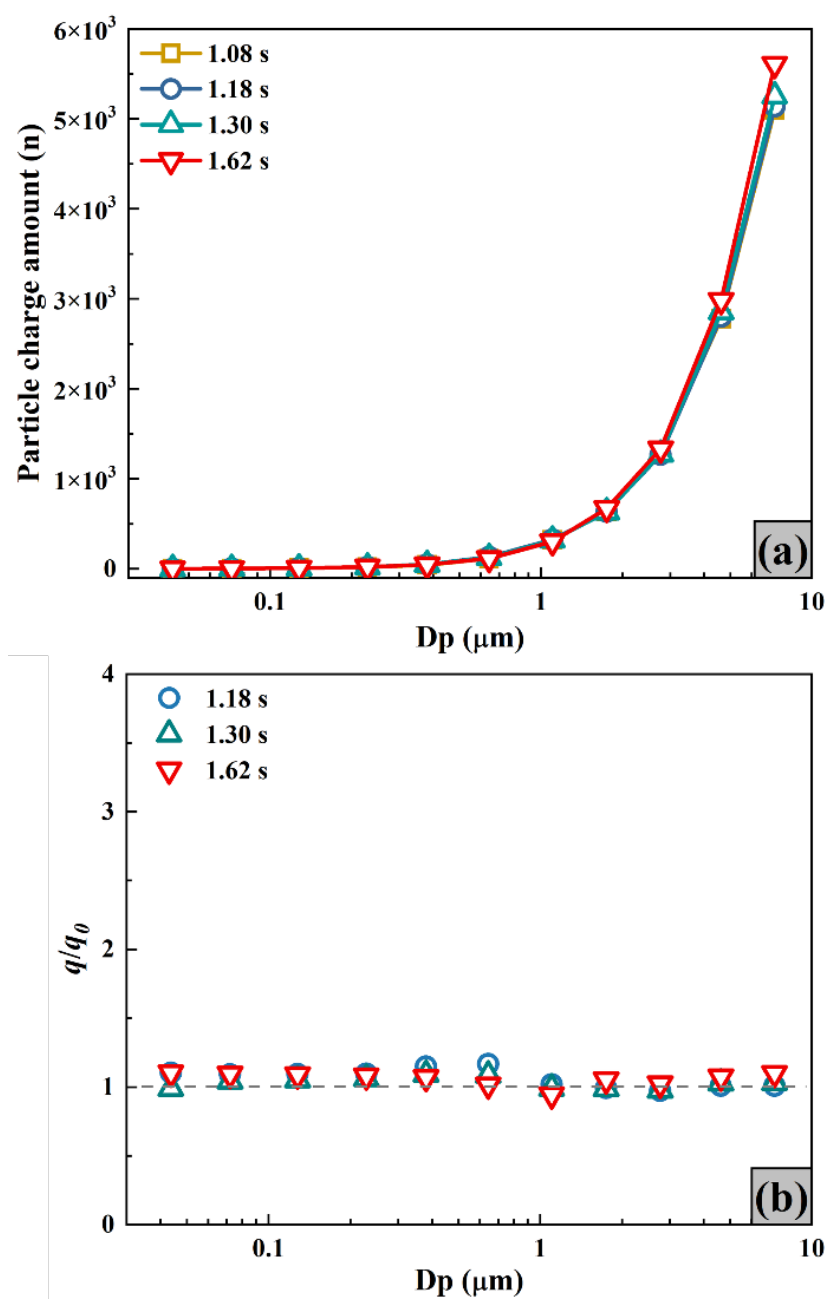

Fig. 7. Charge amount carried by grade particles under various charging time, (a) absolute charge amount, (b) relative charge amount.

charging time is shown in Fig. 7 as a function of particle size. In practical applications, the residence time of flue gas in the WESP is only over one second. Thus the charging time varied in the range of 1.18-1.62 $\mathrm{s}$ in this section. It had been expected to improve the particle charge amount by increasing the charging time. However, the particle charging curves at various charging time almost coincided, as shown in Fig. 7(a). The enhancement amplitude only fluctuated within narrow range, according to Fig. 7 (b) (the data obtained $t=1.08 \mathrm{~s}$ set as a baseline). The fluctuation can even be reasonably considered an experimental error when compared to the data in Fig. 6(b). The results indicated that the particle charging was hardly influenced by charging time in such a small time variation. This provided the potential to develop a compact particle collector with short electric field length from another perspective.

\subsection{Combination of WESP and IME}

The above research was carried out with the mist eliminator disassembled, excluding the contribution of demister to the particle collection. The efficiency data of WESP alone was set as a baseline, and the collection performance of WESP with IME installed was further evaluated. Fig. 8(a) compares the three cases of WESP alone, WESP + one-stage IME, and WESP + two-stage IME. As can be seen, the particle collection efficiency was improved after using the IME. Compared with one-stage IME, the collection efficiency of WESP + two-stage IME could be further increased to $98.9 \%$. At the same time, the charged particle lost charges after impacting through the IME. As shown in Fig. 8(b), the charge loss was $22.6 \%$ and $29.4 \%$ for the particle sized $7.29 \mu \mathrm{m}$ for the one-stage IME and two-stage IME, coinciding with the efficiency enhancement for WESP. 

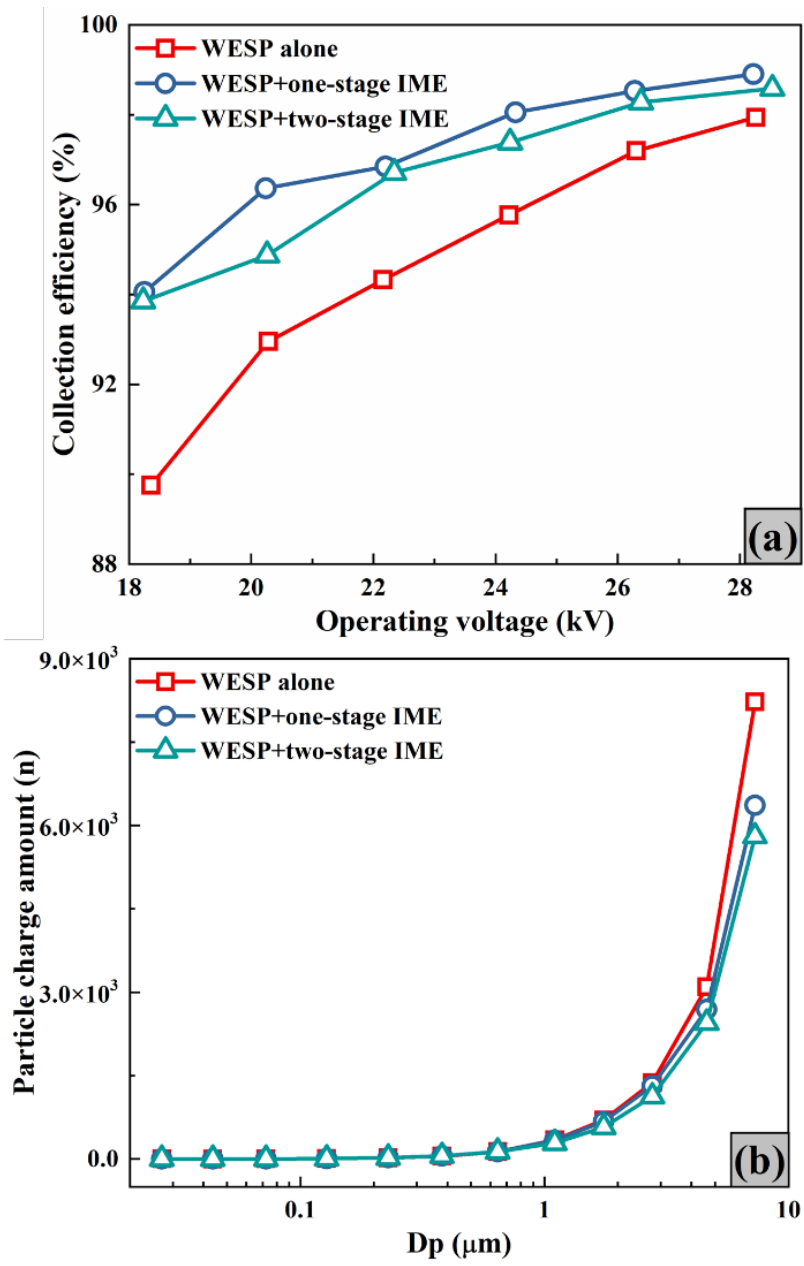

Fig. 8. Performance enhanced by one and two-stage IME, (a) collection efficiency, (b) particle charge amount.

In the WESP, the particle collection is primarily driven by the electric force, while it is inertial impaction contributing to the particle capture in the IME alone. Fig. 9 provides a schematic of the connection between the WESP and IME, which can be analyzed from three aspects. Firstly, the IME surface facing the discharge electrode can act as an additional collection plate of the WESP. Secondly, particles passing through the WESP will incident in a different direction to the mist eliminator, which can change the impaction efficiency (Bernardo et al., 2006). Finally, the impaction efficiency can be improved when external electric fields act on the particles. For the impaction of neutral particles, the capture is primarily driven by inertia and interception (Li et al., 2011). In contrast, the dielectrophoretic force can exert long-range interaction between particles or between each particle and the cylinder for the impaction of polarized particles. Therefore, charged particles can be attracted by dielectrophoretic force over a specific range in space (Li et al., 2011). After impaction, some particles may be captured while some may reflect and still escape. It can be sure that the charge may transfer from the charged particle to the deflection plate during the impaction process (Chen et al., 2014), and Fig. 8(b) can support this analysis as evidence. This result confirms that the combination of WESP and IME for compact designing is possible.

\subsection{Potential of Compact Designing}

Typically, the particle emission concentration can be reduced below $20 \mathrm{mg} \mathrm{m}^{-3}$ after passing through dry ESPs or FFs, which satisfies the Chinese national emission standard of air pollutants for thermal power plants (GB-13223-2011). With the application of WESP, a more stringent ultralow emission level lower than $5 \mathrm{mg} \mathrm{m}^{-3}$ can be achieved since the WESP is easy to achieve a collection efficiency of $>95 \%$. Therefore, it is possible to develop a more compact particle 


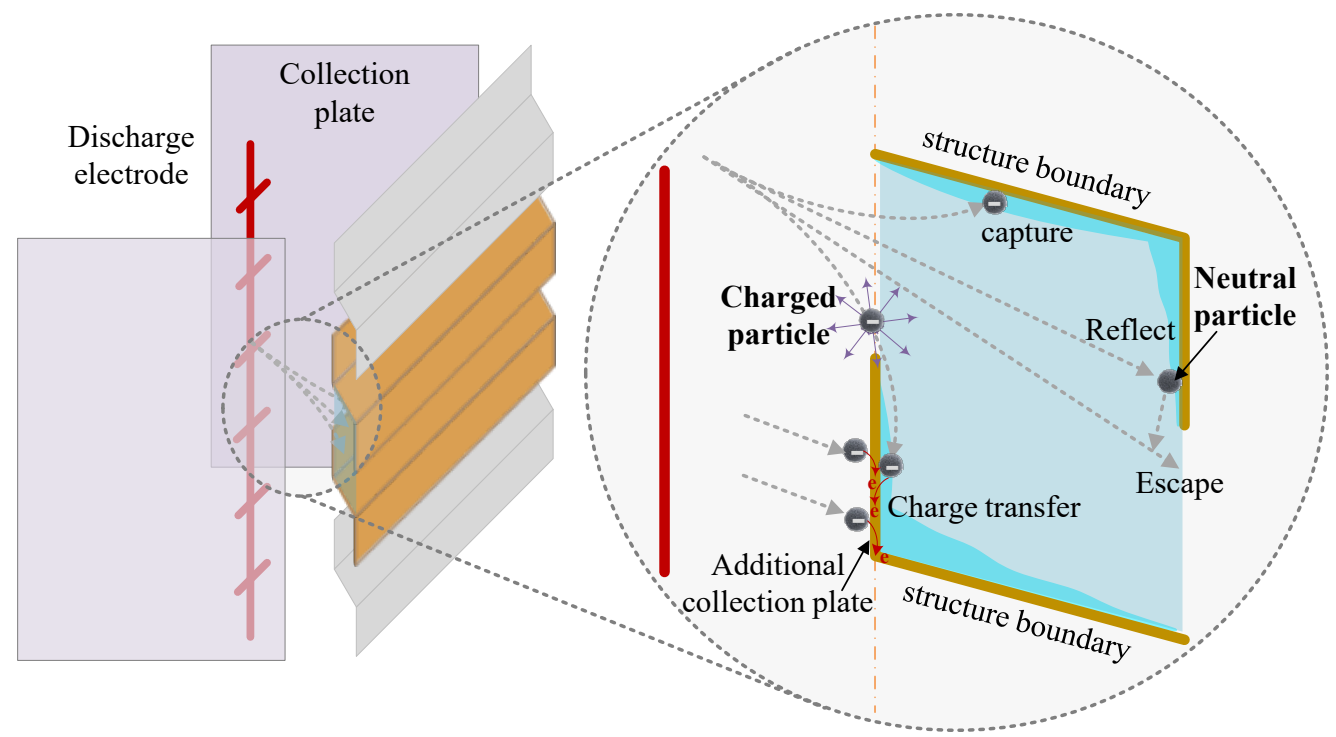

Fig. 9. A schematic of the connection between the WESP and IME.

collector by reducing the WESP size. In the laboratory, the overall dimensions of the WESP are generally fixed, but the effective size can be reduced by removing some electrode wires away. The collection performance of the WESP and IME with various combinations was evaluated under different voltages, as exhibited in Fig. 10(a). The results were further compared in respect with maximum efficiency and specific collection area under the maximum voltage, as exhibited in Fig. 10(b). In this section, the WESP was operated under $3 \times 60 \mathrm{~L} \mathrm{~min}^{-1}$ continuous spraying conditions to better reflect the working conditions, thereby lowering the maximum voltage.

As can be seen, the maximum efficiency of 6 -wire, 8 -wire, and 10 -wire WESP was $88.3 \%, 88.6 \%$, and $98.5 \%$, repectively. Compared with the 10-wire WESP, 8 and 6 wires meant the effective length or specific collection area of WESP decreased by $20 \%$ and $40 \%$. Significantly, the particle collection efficiency was improved when the IME was installed after the length-reduced WESP (6-wire WESP + IME). The collection efficiency of 6-wire WESP enhanced by IME can be even higher than 8-wire WESP alone, ranking the second order in all test cases. Considering both the economy and the performance, the 6-wire WESP + IME can be regarded the optimal combination because it maintained a balance between the size and efficiency. Compared with the 10-wire WESP, the WESP size can be shorten by $40 \%$ while maintaining a similar efficiency.

Although the IME may increase the system resistance, it can significantly reduce the installation space while ensuring collection efficiency. Meanwhile, the combination of WESP with one-stage IME can maintain a better resistance-efficiency balance than that with two-stage IME. Therefore, the development of a compact particle collector was in terms of one-stage IME. In addition to horizontal WESP, the integration of IME is suitable for vertically configured WESP as well. The vertical WESP is usually installed at the top of WFGD. It can be challenging for the device installation and safety when the total height of WFGD and WESP is too high. Therefore, the development of compact vertical WESP also can be beneficial for the PM ultra-low emission reconstruction in cases with insufficient installation space.

\section{CONCLUSIONS}

The development of compact particle collector is of great significance for the PM ultra-low emission in existing units with inadequate retrofit space. In this study, a WESP was designed to achieve a collection efficiency of $95 \%$, and the efficiency can be further improve to $98.9 \%$ after intergrating a IME. The escaping particles through the WESP carried some amounts of charges, which acted as connection between the WESP and IME. The charged particle lost its charges after impacting through the IME. The particle charge loss of the particle-sized $7.29 \mu \mathrm{m}$ was $22.6 \%$ and 29.4\% for the one-stage IME and two-stage IME, coinciding with the efficiency enhancement for 

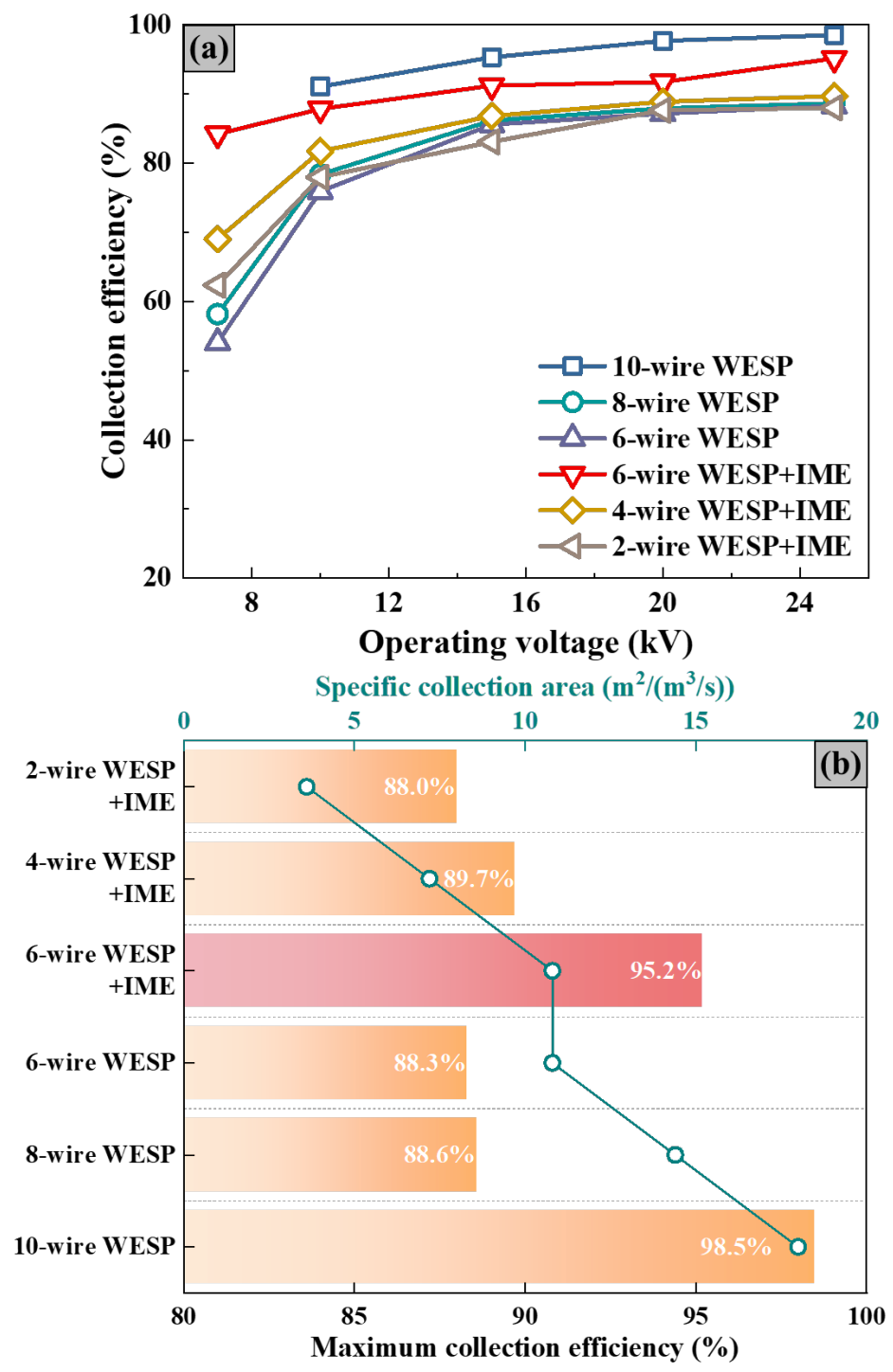

Fig. 10. Particle collection efficiency of WESP with various wires and IME, (a) under different voltages and (b) under maximum voltage.

WESP. Through the IME's enhancement, the WESP size can be reduced to 6 wires while maintaining a collection efficiency higher than that of 8-wire WESP alone. Compared with full size WESP, the 6-wire WESP intergrated with one-stage IME can shorten the WESP size by $40 \%$ while maintaining an efficiency higher than 95\%. In this study, the 6-wire WESP + IME combination can be considered the optimal combination due to its excellent size-efficiency balance.

\section{ACKNOWLEDGMENTS}

This work was supported by the National Natural Science Foundation of China (51906258), Key Research and Development Program of Shandong Province (2019JZZY010403), Qingdao Science and Technology Demonstration and Guidance Project (21-1-4-sf-8-nsh), and Independent Innovation Research Program Projects (21CX06012A).

\section{REFERENCES}

Bernardo, S., Mori, M., Peres, A.P., Dionísio, R.P. (2006). 3-D computational fluid dynamics for gas and gas-particle flows in a cyclone with different inlet section angles. Powder Technol. 162, 
190-200. https://doi.org/10.1016/j.powtec.2005.11.007

Chang, Q., Zheng, C., Yang, Z., Fang, M., Gao, X., Luo, Z., Cen, K. (2017). Electric agglomeration modes of coal-fired fly-ash particles with water droplet humidification. Fuel 200, 134-145. https://doi.org/10.1016/j.fuel.2017.03.033

Chen, B., Li, H., He, Y., Liu, B., Zhang, L. (2019). Study on performance of electrostatic precipitator under multi-physics coupling. Environ. Sci. Pollut. Res. 26, 35023-35033. https://doi.org/10.10 07/s11356-019-06623-8

Chen, S., Li, S., Yang, M., Yao, Q. (2014). Investigation on particles discharging phenomenon in an electrostatic precipitator. J. Eng. Thermophys. 35, 2216-2219.

Cui, L., Li, Y., Tang, Y., Shi, Y., Wang, Q., Yuan, X., Kellett, J. (2018). Integrated assessment of the environmental and economic effects of an ultra-clean flue gas treatment process in coal-fired power plant. J. Cleaner Prod. 199, 359-368. https://doi.org/10.1016/j.jclepro.2018.07.174

Gao, X., Zheng, C., Sagawa, T., Sankar, M., Wu, C., Tronconi, E. (2018). Air pollution control for a green future. J. Zhejiang Univ. Sci. A 19, 1-4. https://doi.org/10.1631/jzus.A17EU001

Huang, S.H., Chen, C.C. (2002). Ultrafine aerosol penetration through electrostatic precipitators. Environ. Sci. Technol. 36, 4625-4632. https://doi.org/10.1021/es011157+

Huang, Y., Zheng, C., Li, Q., Zhang, J., Guo, Y., Zhang, Y., Gao, X. (2020). Numerical simulation of the simultaneous removal of particulate matter in a wet flue gas desulfurization system. Environ. Sci. Pollut. Res. 27, 1598-1607. https://doi.org/10.1007/s11356-019-06773-9

Jiang, Y., Liu, T., Lai, C., Yang, Z., Lin, R., Wang, X., Zhu, X. (2021). Deactivation of $\mathrm{CeO}_{2}-\mathrm{TiO}_{2}$ catalyst by $\mathrm{K}_{2} \mathrm{SO}_{4}$ for $\mathrm{NH}_{3}-\mathrm{SCR}$ : An experimental and DFT study. Appl. Sur. Sci. 547, 149196. https://doi.org/10.1016/j.apsusc.2021.149196

Krupa, A., Podliński, J., Mizeraczyk, J., Jaworek, A. (2019). Velocity field of EHD flow during back corona discharge in electrostatic precipitator. Powder Technol. 344, 475-486. https://doi.org/ 10.1016/j.powtec.2018.12.006

Li, S.Q., Marshall, J.S. (2007). Discrete element simulation of micro-particle deposition on a cylindrical fiber in an array. J. Aerosol Sci. 38, 1031-1046. https://doi.org/10.1016/j.jaerosci.2 007.08 .004

Li, S., Marshall, J.S., Liu, G., Yao, Q. (2011). Adhesive particulate flow: The discrete-element method and its application in energy and environmental engineering. Prog. Energy Combust. Sci. 37, 633-668. https://doi.org/10.1016/j.pecs.2011.02.001

Li, X., Zhou, C., Li, J., Lu, S., Yan, J. (2019). Distribution and emission characteristics of filterable and condensable particulate matter before and after a low-low temperature electrostatic precipitator. Environ. Sci. Pollut. Res. 26, 12798-12806. https://doi.org/10.1007/s11356-01904570-y

Liang, Y., Li, Q., Ding, X., Wu, D., Wang, F., Otsuki, T., Cheng, Y., Shen, T., Li, S., Chen, J. (2020). Forward ultra-low emission for power plants via wet electrostatic precipitators and newly developed demisters: Filterable and condensable particulate matters. Atmos. Environ. 225, 117372. https://doi.org/10.1016/j.atmosenv.2020.117372

Liu, Y., Hu, B., Zhou, L., Jiang, Y., Yang, L. (2016). Improving the removal of fine particles with an electrostatic precipitator by chemical agglomeration. Energy Fuels 30, 8441-8447. https://doi.org/10.1021/acs.energyfuels.6b00626

Lu, Q., Yang, Z., Zheng, C., Li, X., Zhao, C., Xu, X., Gao, X., Luo, Z., Ni, M., Cen, K. (2016). Numerical simulation on the fine particle charging and transport behaviors in a wire-plate electrostatic precipitator. Adv. Powder Technol. 27, 1905-1911. https://doi.org/10.1016/j.apt.2016.06.021

Mizuno, A. (2000). Electrostatic precipitation. IEEE Trans. Dielect. Electr. Insul. 7, 615-624. https://doi.org/10.1109/94.879357

Shuangchen, M., Jin, C., Gongda, C., Weijing, Y., Sijie, Z. (2016). Research on desulfurization wastewater evaporation: Present and future perspectives. Renewable Sustainable Energy Rev. 58, 1143-1151. https://doi.org/10.1016/j.rser.2015.12.252

Song, J., Hu, X. (2017). A mathematical model to calculate the separation efficiency of streamlined plate gas-liquid separator. Sep. Purif. Technol. 178, 242-252. https://doi.org/10.1016/j.seppur. 2017.01.044

Wang, B., Liu, H., Zhou, C., Huo, H., Dong, K., Jiang, Y. (2020). Enhancing the collection efficiency of a gas cyclone with atomization and electrostatic charging. Powder Technol. 364, 562-571. https://doi.org/10.1016/j.powtec.2020.01.062 
Xu, Y., Liu, X., Cui, J., Chen, D., Xu, M., Pan, S., Zhang, K., Gao, X. (2016). Field measurements on the emission and removal of $\mathrm{PM}_{2.5}$ from coal-fired power stations: 4. PM removal performance of wet electrostatic precipitators. Energy Fuels 30, 7465-7473. https://doi.org/10.1021/acs.en ergyfuels.6b00426

Yang, Z., Zheng, C., Chang, Q., Wan, Y., Wang, Y., Gao, X., Cen, K. (2017). Fine particle migration and collection in a wet electrostatic precipitator. J. Air Waste Manage. Assoc. 67, 498-506. https://doi.org/10.1080/10962247.2016.1260074

Yang, Z., Zheng, C., Liu, S., Guo, Y., Liang, C., Wang, Y., Hu, D., Gao, X. (2018a). A combined wet electrostatic precipitator for efficiently eliminating fine particle penetration. Fuel Process. Technol. 180, 122-129. https://doi.org/10.1016/j.fuproc.2018.08.013

Yang, Z., Zheng, C., Zhang, X., Chang, Q., Weng, W., Wang, Y., Gao, X. (2018b). Highly efficient removal of sulfuric acid aerosol by a combined wet electrostatic precipitator. RSC Adv. 8, 5966. https://doi.org/10.1039/C7RA11520B

Yang, Z., Zheng, C., Liu, S., Guo, Y., Liang, C., Zhang, X., Zhang, Y., Gao, X. (2018c). Insights into the role of particle space charge effects in particle precipitation processes in electrostatic precipitator. Powder Technol. 339, 606-614. https://doi.org/10.1016/j.powtec.2018.08.056

Yang, Z., Ji, P., Li, Q., Jiang, Y., Zheng, C., Wang, Y., Gao, X., Lin, R. (2019). Comprehensive understanding of $\mathrm{SO}_{3}$ effects on synergies among air pollution control devices in ultra-low emission power plants burning high-sulfur coal. J. Cleaner Prod. 239, 118096. https://doi.org/ 10.1016/j.jclepro.2019.118096

Yang, Z., Zheng, C., Li, Q., Zheng, H., Zhao, H., Gao, X. (2020a). Fast evolution of sulfuric acid aerosol activated by external fields for enhanced emission control. Environ. Sci. Technol. 54, 3022-3031. https://doi.org/10.1021/acs.est.9b06191

Yang, Z., Li, H., Li, Q., Lin, R., Jiang, Y., Yang, Y., Zheng, C., Sun, D., Gao, X. (2020b). Correlations between particle collection behaviors and electrohydrodynamics flow characteristics in electrostatic precipitators. Aerosol Air Qual. Res. 20, 2901-2910. https://doi.org/10.4209/aaq r.2020.07.0475

Yang, Z., Cai, Y., Li, Q., Li, H., Jiang, Y., Lin, R., Zheng, C., Sun, D., Gao, X. (2021). Predicting particle collection performance of a wet electrostatic precipitator under varied conditions with artificial neural networks. Powder Technol. 377, 632-639. https://doi.org/10.1016/j.powtec.2020.09.027

Ye, C., Zheng, Y., Xu, Y., Li, G., Dong, C., Tang, Y., Wang, Q. (2020). Energy and exergy analysis of poly-generation system of hydrogen and electricity via coal partial gasification. Comput. Chem. Eng. 141, 106911. https://doi.org/10.1016/j.compchemeng.2020.106911

Zheng, C., Wang, Y., Liu, Y., Yang, Z., Qu, R., Ye, D., Liang, C., Liu, S., Gao, X. (2019). Formation, transformation, measurement, and control of $\mathrm{SO}_{3}$ in coal-fired power plants. Fuel 241, 327346. https://doi.org/10.1016/j.fuel.2018.12.039

Zhou, D., Luo, Z., Jiang, J., Chen, H., Lu, M., Fang, M. (2016). Experimental study on improving the efficiency of dust removers by using acoustic agglomeration as pretreatment. Powder Technol. 289, 52-59. https://doi.org/10.1016/j.powtec.2015.11.009 